\title{
A tribute to the memory of Pavel Evseevich Sobolevskii (1930-2018)
}

\author{
Allaberen Ashyralyev and Abdullah S. Erdogan \\ Communicated by M. Zuhair Nashed
}

\begin{abstract}
This tribute is devoted to Pavel Evseevich Sobolevskii's lovely memory and gives a summary of his important contributions to mathematics.
\end{abstract}

\section{Pavel Evseevich Sobolevskii}

On August 16, 2018, we were deeply shocked and saddened to hear the loss of our Honorary Editor Pavel Evseevich Sobolevskii. Professor Pavel Evseevich Sobolevskii passed away at age 88 in Fortaleza, Brazil surrounded by his family. He was a well known expert on partial differential equations (PDEs), functional analysis and numerical analysis. He will be truly missed by our Editorial Board, his friends and family. It is a fact that we lost him, but we did not lose him as a model in our life, his devotion and strength. This tribute is devoted to his lovely memory and gives a summary of his important contributions to mathematics.

Professor Sobolevskii was born on March 26, 1930 in Kiev, Ukraine. After receiving his undergraduate degree from Kiev State University, he entered graduate school in Voronezh, Russia, where a major school in functional analysis was being created at the time. Two giants of this school, M. A. Krasnosel'skii and S. G. Krein were advisors on his Ph.D. dissertation, defended in 1958. As a part of his thesis, Sobolevskii introduced and used masterfully the notion of an "acute angle" between operators, which enables one to compare complex operators with simpler ones. This technique has been used later by many mathematicians, e.g., J. L. Lions, H. Brezis, and L. Nirenberg. The intertwining themes of functional analysis and PDEs had been underlying Pavel's research throughout his distinguish mathematical career.

Starting in 1958, Sobolevskii worked at the Department of Mathematics of the Voronezh Agriculture Institute, chairing the department from 1964 to 1968. In 1962, he received the prestigious and hard to get Doctor of Science degree (an upscale version of European Habilitation). In 1968, when M. A. Krasnosel'skii moved to Moscow, P. E. Sobolevskii became the Head of the Department of Func- 
tional Analysis and Operator Equations at Voronezh State University. Among many other (besides the "acute angle") scientific achievements of P. E. Sobolevskii, one can mention his joint work with V. Maz'ya for the proof of the classical and important criterion of existence of a contracting operator semigroup in a Banach space with differentiable norm (this result is usually attributed to Lumer and Phillips, although Sobolevskii and Maz'ya obtained it simultaneously and independently); development in the context of analytic semigroups of the so called Kato-Sobolevskii-Tanabe theory of non-autonomous parabolic equations; important results on parabolic equations, which in particular implied one of the first local existence theorems for the Navier-Stokes equation; development of the theory of fractional powers of positive operators; new methods of numerical solutions of PDEs, and a lot more.

Sobolevskii was a prolific and well cited author, publishing around 180 research articles and four monographs on PDEs, functional analysis, and numerical methods. Besides leading a very active research, he was also an accomplished teacher and advisor. He has an advisor on 35 Ph.D. dissertations. Math Genealogy Project lists 67 of his "research descendants". In 1991, P. E. Sobolevskii moved to Israel, where he worked at the Hebrew University till his retirement in 2003. After that he moved to Brazil to join his daughter's family. From 2003 till 2006 he held a visiting position at the Universidade Federal do Ceara.

The memory of Pavel Evseevich Sobolevskii, a scientist, a scholar, and a remarkable person, will live in his works and in the hearts of his family, students, and colleagues.

\section{Sobolevskii's important selected results}

Pavel E. Sobolevskii is best known for his following contributions:

\subsection{On equations with operators forming an acute angle}

One of the commonly used techniques for studying complex equations is that these equations are compared with simpler ones, whose properties are known. Moreover, for the compared two equations several common properties follow (solvability of equations, the possibility of using approximate methods, etc).

For the first time in paper [1] Sobolevskii proposed a simple principle of comparison of two operators, which allows one to get several new approaches on elliptic and parabolic equations.

1. Definition. The linear operators $A$ and $B$ acting in the Hilbert space $H$ form an acute angle if they have a common domain $D=D(A)=D(B)$, vanish only at zero, and if there is a positive number $m$ such that 


$$
(A x, B x) \geq m\|A x\| \cdot\|B x\| \quad(x \in D) .
$$

An elementary calculation shows that the solution in the sense of Krasnosel'skii and Krein between the linear sets $R(A)$ and $R(B)$, the values of the operators $A$ and $B$ forming an acute angle do not exceed $1-m$. This yields the following result.

Theorem 2.1. Let the operators $A$ and $B$ form an acute angle. Then they have the same defect indices, more precisely, the orthogonal complements of $\overline{R(A)}$ and $\overline{R(B)}$ have the same dimension.

2. Let $\left\{R_{n}\right\}$ be a monotonically increasing sequence of subspaces of space $H$ and $\bigcup_{n=1}^{\infty} R_{n}=H$. Denote by $P_{n}$ the orthogonal projection operator on $R_{n}$. For an approximate solution of the equation $B x=f$, the solution $x_{n}$ of "approximate" equations $P_{n} B x=P_{n} f$ can be sought.

Theorem 2.2. Let the operators $A$ and $B$ form an acute angle and $R(A)=$ $R(B)=H$. Let the subspaces $R_{n}$ be invariant under the self-adjoint operator $A$. Then for each $n$ the equation $P_{n} B x=P_{n} f$ has a solution $x_{n} \in R_{n}$. Approximate solutions $x_{n}$ as $n \rightarrow \infty$ converge in norm to the solution $x$ of equation $B x=f$, and the norms of residuals $B x_{n}-f$ tend to zero.

Consider the initial value problem

$$
\frac{d x}{d t}+B x(t)=0, t>0, x(0)=x_{0}\left(x_{0} \in D(B)\right) .
$$

Consider also the approximate solution $x_{n}(t)$ of problem (2), more precisely, the solution of the initial value problem:

$$
\frac{d x}{d t}+P_{n} B x(t)=0, t>0, x_{n}(0)=P_{n} x_{0} .
$$

Theorem 2.3. Let the conditions of Theorem 2.2 be satisfied and let for each $x \in$ $D(B)$ the following inequalities hold:

$$
(A x, x) \geq(x, x),(B x, x) \geq(x, x) .
$$

Then problems (2) and (3) are solvable, moreover, for each $T>0$

$$
\lim _{n \rightarrow \infty} \max _{t \in[0, T]}\left\|x_{n}(t)-x(t)\right\|=0,
$$




$$
\lim _{n \rightarrow \infty} \max _{t \in[0, T]}\left\|\frac{d x_{n}(t)}{d t}+B x_{n}(t)\right\|=0,
$$

where $x(t)$ is the solution of problem (2).

3. Definition. Let $A$ be a linear with dense domain in a Hilbert space $H$ and with range $R(A)$ in a Hilbert space $H_{1}$. Then $A$ is normally solvable if $\overline{R(A)}=$ $R(A)$.

Let $A^{*}$ be the adjoint of $A$. For it to be normally solvable it is necessary and sufficient that $R(A)=^{\perp} N\left(A^{*}\right)$, that is, the range of $A$ is the orthogonal complement to the null space of $A^{*}$.

Let the operators $A$ and $B$ be normally solvable and form an acute angle. It is easy to see that perturbed operators $A+F_{1}$ and $B+F_{2}$ also form an acute angle, if the operators $F_{1}$ and $F_{2}$ are bounded and have sufficiently small norms. In some cases, it is possible to show that the acuteness of the angle is also preserved under unbounded perturbations.

Theorem 2.4. Let $A$ and $B$ be positive definite self-adjoint operators with a common domain D. Let constants $\alpha_{1}, \alpha_{2}$ and $m$ characterize the comparability of these operators and the angle between them:

$$
\alpha_{1}\|A x\| \leq\|B x\| \leq \alpha_{2}\|A x\|,(A x, B x) \geq m\|A x\| \cdot\|B x\|(x \in D) .
$$

Finally, let the operator $F$ defined on D satisfy the inequality

$$
\|F x\|^{2} \leq \delta^{2}\|A x\|^{2}+c^{2}\left\|A^{1-\varepsilon} x\right\|^{2} \quad(x \in D)
$$

for some $c>0, \varepsilon>0$ and $0<\delta<\alpha_{1} m$. Then for sufficiently large $k$ the operator $B+F+k I$ is normally solvable and forms an acute angle with the operator $A$.

In particular, the statement of Theorem 2.4 is true for the operator $B+F+k I$, if $0<\delta<1$. Therefore, if the operator $A$ has a completely continuous inverse, then $A+F$ is a Fredholm operator.

4. We denote by $H_{1}$ Hilbert space of Bochner integrable functions on $[0, T]$ with values in $H_{1}$. The scalar product in $H_{1}$ is defined by

$$
[x, y]_{H_{1}}=\int_{0}^{T}(x(t), y(t)) d t .
$$

Let $A$ be a positive definite self-adjoint operator with the domain $D$. The operator $d / d t+A$ is directly determined on such continuously differentiable functions 
$x(t) \in H_{1}$, that the functions $A x(t)$ are continuous and $x(0)=0$. The closure of this operator in $H_{1}$ is also denoted by $d / d t+A$ (we can show that this closure exists). The domain of the constructed closure is denoted by the operator $S$.

It is well-known that the equation

$$
\frac{d x}{d t}+A x=f(t)
$$

considered in $H_{1}$ has a solution $x(t) \in S$, if $f(t) \in H_{1}$.

Theorem 2.5. Let the operators $A(t), B(t)$ and $F(t)$ for each $t \in[0, T]$ be defined on $D$ and satisfy the conditions of Theorem 2.4 with constants $\alpha_{1}>0, \alpha_{2}>0$, $m>0, c>0, \varepsilon>0$ and $0<\delta<2 \sqrt{\alpha_{1} m+1}-2$, independent of $t$. Suppose that for each $x \in D$ the functions $A(t) x, B(t) x$ are continuous, piecewise continuously differentiable, and the function $F(t) x$ is continuous in $t$. Then, for sufficiently large $k$, the operator $d / d t+B(t)+F(t)+k I$ acting on $H_{1}$ defined on $S$ is normally solvable and forms an acute angle with the operator $d / d t+A(t)$.

In particular, the statement of Theorem 2.5 is true for the operator

$$
d / d t+A(t)+F(t)+k I,
$$

if $0<\delta<\frac{4}{5}$.

5. We turn to the consideration of specific differential operators.

We denote by $\bar{\Omega}$ a closed bounded region of $n$ dimensional space. Let $W_{2}^{2}$, as usual, denote the closure of $W_{2}^{2}$ in the norm

$$
\|u\|_{W_{2}^{2}}^{2}=\int_{\Omega}\left[u^{2}+\sum_{i=1}^{n}\left(\frac{\partial u}{\partial x_{i}}\right)^{2}+\sum_{i, k=1}^{n}\left(\frac{\partial^{2} u}{\partial x_{i} \partial x_{k}}\right)^{2}\right] d x
$$

be sets of thrice continuously differentiable functions in $\bar{\Omega}$ that vanish on the boundary. In $L_{2}(\Omega)$ we consider differential operators

$$
\begin{gathered}
A \equiv-\sum_{i, k=1}^{n} a_{i k}(x) \frac{\partial^{2}}{\partial x_{i} \partial x_{k}}+\sum_{i=1}^{n} a_{i}(x) \frac{\partial}{\partial x_{i}}+a(x) I, \\
B \equiv-\sum_{i, k=1}^{n} b_{i k}(x) \frac{\partial^{2}}{\partial x_{i} \partial x_{k}}+\sum_{i=1}^{n} b_{i}(x) \frac{\partial}{\partial x_{i}}+b(x) I
\end{gathered}
$$

with domain $\stackrel{\circ}{W_{2}^{2}}$. We assume that on $\bar{\Omega}$ the functions $a_{i k}$ and $b_{i k}$ are continuously differentiable, the functions $a_{i}, b_{i}, a$ and $b$ are continuous and forms

$$
\sum_{i, k=1}^{n} a_{i k}(x) \xi_{i} \xi_{k} \text { and } \sum_{i, k=1}^{n} b_{i k}(x) \xi_{i} \xi_{k}
$$

are positive definite for each $x \in \bar{\Omega}$. 
Theorem 2.6. There exist positive numbers $m>0, n>0$, such that for all functions $u(x) \in W_{2}^{2}$ the following inequality holds

$$
\begin{aligned}
\int_{\Omega} A u \cdot B u d x \geq & m \int_{\Omega} \sum_{i, k=1}^{n}\left(\frac{\partial^{2} u}{\partial x_{i} \partial x_{k}}\right)^{2} d x \\
& -n \int_{\Omega}\left[\sum_{i=1}^{n}\left(\frac{\partial u}{\partial x_{i}}\right)^{2}+u^{2}\right] d x .
\end{aligned}
$$

If the function $a(x)$ is bounded below by a sufficiently large number depending only on functions $a_{i k}$ and $a_{i}$, then for setting $B=A$ in (12) we obtain the wellknown inequality of Ladyzhenskaya

$$
\|A u\|_{L_{2}}^{2} \geq c^{2}\|u\|_{W_{2}^{2}}^{2}\left(u \in \stackrel{\circ}{W_{2}^{2}}\right) .
$$

Moreover, under the same condition, the operator $A$ forms an acute angle with the operator $B+k I$, where $k$ is a sufficiently large number.

6. Let $H=L_{2}(\Omega)$ and let $\Delta$ be the Laplace operator defined on $W_{2}^{2}$. For selfadjoint positive definite operator $-\Delta$ we construct the operator $d / d t-\Delta$ acting on $H_{1}$. Its domain is denoted by $S$. The next theorem follows from Theorem 2.5:

Theorem 2.7. Assume that the operator

$$
A(t) \equiv-\sum_{i, k=1}^{n} a_{i k}(t, x) \frac{\partial^{2}}{\partial x_{i} \partial x_{k}}+\sum_{i=1}^{n} a_{i}(t, x) \frac{\partial}{\partial x_{i}}+a(t, x) I
$$

for each $t \in[0, T]$ satisfies the conditions of item 5 above. Let the functions $a_{i k}(t, x)$ be continuous, piecewise continuously differentiable, and the functions $a_{i}(t, x)$ and $a(t, x)$ be continuous in $t$. Then, for a sufficiently large $k$ the operator $d / d t+A(t)+k I$ acting on $H_{1}$ is normally solvable and forms an acute angle with the operator $d / d t-\Delta$.

7. Applying Theorem 2.1 to the operators considered in items 4-6, we obtain statements on the solvability of various equations [2]. Let us give examples.

If the operators $A(t)$ and $F(t)$ satisfy the conditions of item $\mathbf{4}$, then the equation

$$
\frac{d x}{d t}+A(t) x+F(t) x=f(t)
$$

has a solution $x(t) \in S$, if $f(t) \in H_{1}$. This statement remains valid under restrictions on the operator $A(t)$ weaker than piecewise differentiability. It is enough to 
require that the operator $A(t) A^{-1}(0)$ be continuous in operator norm. This statement was proved by approximating the operator $A(t)$ with a special way selecting continuous, piecewise continuously differentiable operators. In particular, this implies solvability of parabolic equations with a second order elliptic operator with zero boundary conditions, if the coefficients of this operator continuously depend on $t$. The above statements generalize some theorems of Ladyzhenskaya on the solvability of the boundary-value problem for the elliptic equation $A u=f$.

8. Application of Theorems 2.2 and 2.3 to the differential equations considered above to operators (item 5) gives theorems on the convergence of the BubnovGalerkin method for elliptic and parabolic equations. In addition to the convergence of the approximations to the solution, there is convergence of the residuals to zero. This fact for self-adjoint elliptic equations was previously established by Mikhlin. We denote by $P_{n}$ orthogonal projection operator on linear shell $R_{n}$ of the first $n$ elements of the basis $\left\{e_{j}\right\}$, consisting of eigenvectors of some self-adjoint elliptic operator.

Theorem 2.8. Let the operator A satisfy the conditions of item 5 . Then, for every $n$ there is a solution $u_{n} \in R_{n}$ of equations $P_{n} A u_{n}=P_{n} f$. Moreover, as $n \rightarrow \infty$ solutions $u_{n}$ converge in norm to the solution of the equation $A u=f$, and the norms of residuals $A u_{n}-f$ tend to zero.

Theorem 2.9. Let the operator A satisfy the conditions of item 5. Then, for every $n$ there is a solution $u_{n}(t)$ of the initial value problem

$$
\frac{d u}{d t}+P_{n} A u=0, u_{n}(0)=P_{n} u_{0}\left(u_{0} \in \stackrel{\circ}{W_{2}^{2}}\right) .
$$

Furthermore, as $n \rightarrow \infty$ the solutions $u_{n}(t)$ converge in norm, uniformly in $t \in$ $[0, T]$ to the solution of the $u(t)$ of the problem

$$
\frac{d u}{d t}+A u=0, u(0)=u_{0},
$$

and the norms of discrepancies $\frac{d u_{n}}{d t}+A u_{n}$ tend to zero.

\subsection{Coerciveness inequalities for abstract parabolic equations}

The role played by coercive inequalities in the study of boundary value problems for elliptic and parabolic partial differential equations is well known. Coercivity inequalities for the solutions of an Cauchy problem for abstract differential equations of parabolic type 


$$
v^{\prime}(t)+A(t) v(t)=f(t)(0 \leq t \leq T), v(0)=v_{0}
$$

in an arbitrary Banach space $E$ with the linear (unbounded) operators $A(t)$ were established for the first time by Sobolevskii in the paper [3]. Here $v(t)$ and $f(t)$ are the unknown and the given functions, respectively, defined on $[0, T]$ with values in $E$. The derivative $v^{\prime}(t)$ is understood as the limit in the norm of $E$ of the corresponding ratio of differences. $A(t)$ is a given, closed, linear operator with domain $D(A(t))=D$, independent of $t$ and dense in $E$. Finally, $v_{0}$ is a given element of $E$.

A function $v(t)$ is called a solution of problem (14) if the following conditions are satisfied:

i. $v(t)$ is continuously differentiable on the segment $[0, T]$. The derivative at the endpoints of the segment are understood as the appropriate unilateral derivatives.

ii. The element $v(t)$ belongs to $D$ for all $t \in[0, T]$, and the function $A(t) v(t)$ is continuous on $[0, T]$.

iii. $v(t)$ satisfies the equation and the initial condition (14).

A solution of problem (14) defined in this manner will from now on be referred to as a solution of problem (14) in the space $C(E)=C([0, T], E)$. Here, $C(E)$ stands for the Banach space of all continuous functions $\varphi(t)$ defined on $[0, T]$ with values in $E$ equipped with the norm

$$
\|\varphi\|_{C(E)}=\max _{0 \leq t \leq T}\|\varphi(t)\|_{E} .
$$

From the existence of the such solutions, it follows that $f(t) \in C(E)$ and $v_{0} \in D$.

We say that problem (14) is well-posed in $C(E)$, if the following conditions are satisfied:

1. Problem (14) is uniquely solvable for any $f(t) \in C(E)$ and any $v_{0} \in D$. This induces an additive and homogeneous operator via $v(t)=v\left(t ; f(t), v_{0}\right)$ which acts from $C(E) \times D$ to $C(E)$ and gives the solution of problem (14) in $C(E)$.

2. $v\left(t ; f(t), v_{0}\right)$, regarded as an operator from $C(E) \times D$ to $C(E)$, is continuous. Here, $C(E) \times D$ is understood as the normed space of the pairs $\left(f(t), v_{0}\right), f(t) \in C(E)$ and $v_{0} \in D(A)$, equipped with the norm

$$
\left\|\left(f(t), v_{0}\right)\right\|_{C(E) \times D}=\|f\|_{C(E)}+\left\|v_{0}\right\|_{D} .
$$


Following the well-known result due to Banach in $C(E)$ [36, p. 100] and these properties one has the coercive inequality

$$
\left\|v^{\prime}\right\|_{C(E)}+\|A(.) v\|_{C(E)} \leq M_{C}\left[\|f\|_{C(E)}+\left\|v_{0}\right\|_{D}\right]
$$

where $M_{C}\left(1 \leq M_{C}<\infty\right)$ does not depend on $v_{0}$ and $f(t)$.

This inequality is called the coercivity inequality in $C(E)$ for (14). If $A(t)=A$, then the coercivity inequality implies the analyticity of the semigroup $\exp \{-s A\}$ $(s \geq 0)$ for all $t$, i.e., the following estimates

$$
\|\exp (-s A)\|_{E \rightarrow E},\|s A \exp (-s A)\|_{E \rightarrow E} \leq M(s>0)
$$

hold for some $M \in[1, \infty)$. Thus, the analyticity of the semigroup $\exp \{-s A\}$ $(s \geq 0)$ is necessary for the well-posedness of problem (14) in $C(E)$. Unfortunately, the analyticity of the semigroup $\exp \{-s A\}(s \geq 0)$ is not a sufficient for the well-posedness of problem (14) in $C(E)$.

Suppose that for each $t \epsilon[0, T]$ the operator $-A(t)$ generates an analytic semigroup $\exp \{-s A(t)\}(s \geq 0)$ with exponentially decreasing norm, when $s \longrightarrow \infty$, i.e., the following estimates

$$
\|\exp (-s A(t))\|_{E \rightarrow E},\|s A(t) \exp (-s A(t))\|_{E \rightarrow E} \leq M e^{-\delta s}(s>0)
$$

hold for some $M \in[1, \infty), \delta \in(0, \infty)$. From this inequality it follows that the operator $A^{-1}(t)$ exists and is bounded; hence $A(t)$ is closed in $C(E)$.

Suppose that the operator $A(t) A^{-1}(s)$ is Hölder continuous in $t$ in the uniform operator topology for each fixed $s$, that is,

$$
\left\|[A(t)-A(\tau)] A^{-1}(s)\right\|_{E \rightarrow E} \leq M|t-\tau|^{\varepsilon}, 0<\varepsilon \leq 1,
$$

where $M$ and $\varepsilon$ are positive constants independent of $t, s$ and $\tau$ for $0 \leq t, s, \tau \leq T$.

An operator-valued function $v(t, s)$, defined and strongly continuous jointly in $t, s$ for $0 \leq s<t \leq T$, is called a fundamental solution of (14), if

1) the operator $v(t, s)$ is strongly continuous in $t$ and $s$ for $0 \leq s<t \leq T$,

2) the following identity holds:

$$
v(t, s)=v(t, \tau) v(\tau, s), v(t, t)=I \text { for } 0 \leq s \leq \tau \leq t \leq T,
$$

3) the operator $v(t, s)$ maps the region $D$ into itself. The operator $u(t, s)=$ $A t) v(t, s) A^{-1}(s)$ is bounded and strongly continuous in $t$ and $s$ for $0 \leq s<t \leq T$, 
4) on the region $D$ the operator $v(t, s)$ is strongly differentiable with respect to $t$ and $s$ with

$$
\frac{\vartheta v(t, s)}{\vartheta t}=-A(t) v(t, s)
$$

and

$$
\frac{\vartheta v(t, s)}{\vartheta s}=v(t, s) A(s) .
$$

$v(t, s)$ is also called evolution operator, Green's function, etc.

If the function $f(t)$ is not only continuous but also continuously differentiable on $[0, T]$, and $v_{0} \in D$, it is easy to show that the formula

$$
v(t)=v(t, 0) v_{0}+\int_{0}^{t} v(t, s) f(s) d s
$$

gives a solution of problem (14).

The Cauchy problem (14) has been considered in various spaces $F(E)$ of functions defined on the segment $[0, T]$ with values in a Banach space $E$.

A function $v(t)$ is said to be a solution of problem (14) in $F(E)$, if it is a solution of this problem in $C(E)$ and the function $v^{\prime}(t)$ and $A(t) v(t)$ belong to $F(E)$.

As in the case of the space $C(E)$, we say that problem (14) is well-posed in $F(E)$, if the following two conditions are satisfied:

1. For any $f \in F(E)$ and $v_{0} \in D(A(t))$ there exists the unique solution $v(t)=$ $v\left(t ; f(t), v_{0}\right)$ in $F(E)$ of problem (14).This means that an additive and homogeneous operator $v\left(t ; f(t), v_{0}\right)$ is defined which acts from $F(E) \times D(A(t))$ to $F(E)$ and gives the solution of (14) in $F(E)$.

2. $v\left(t ; f(t), v_{0}\right)$, regarded as an operator from $F(E) \times D(A(t))$ to $F(E)$, is continuous.Here $F(E) \times D(A(t))$ is understood as the normed space of the pairs $\left(f(t), v_{0}\right), f(t) \in F(E)$ and $v_{0} \in D(A(t))$, equipped with the norm

$$
\left\|\left(f(t), v_{0}\right)\right\|_{F(E) \times D(A(t))}=\|f\|_{F(E)}+\left\|v_{0}\right\|_{D(A(t))} .
$$

We set $F(E)$ to be $C_{0}^{\alpha, \alpha}(E)=C_{0}^{\alpha, \alpha}([0, T], E), 0<\alpha<1$, the Banach space obtained by completion of the set of smooth $E$-valued functions $\varphi(t)$ on $[0, T]$ in the norm

$$
\|\varphi\|_{C_{0}^{\alpha, \alpha}(E)}=\|\varphi\|_{C(E)}+\sup _{0 \leq t<t+\tau \leq T} \frac{\|\varphi(t+\tau)-\varphi(t)\|_{E}}{\tau^{\alpha}}(t+\tau)^{\alpha} .
$$

As in the case of the space $C(E)$, from the well-posedness of the Cauchy problem (14) one derives the coercivity inequality

$$
\left\|v^{\prime}\right\|_{C_{0}^{\alpha, \alpha}(E)}+\|A(.) v\|_{C_{0}^{\alpha, \alpha}(E)} \leq M_{C}(\alpha)\left[\|f\|_{C_{0}^{\alpha, \alpha}(E)}+\left\|A(0) v_{0}\right\|_{E}\right],
$$


where $M_{C}(\alpha)\left(1 \leq M_{C}(\alpha)<\infty\right)$ does not depend on $v_{0}$ and $f(t)$.

Theorem 2.10. Suppose $v_{0} \in D(A(t)), f(t) \in C_{0}^{\alpha, \alpha}(E)$. Suppose that the assumptions (16) and (17) hold and $0<\alpha \leq \varepsilon<1$. Then, the Cauchy problem (14) is well-posed in Hölder space $C_{0}^{\alpha, \alpha}(E)$. Moreover, for the solution $v(t)$ in $C_{0}^{\alpha, \alpha}(E)$ of the Cauchy problem the coercive inequality

$$
\left\|v^{\prime}\right\|_{C_{0}^{\alpha, \alpha}(E)}+\|A(.) v\|_{C_{0}^{\alpha, \alpha}(E)} \leq \frac{M}{\alpha(1-\alpha)}\|f\|_{C_{0}^{\alpha, \alpha}(E)}+M\left\|A(0) v_{0}\right\|_{E}
$$

holds, where $M$ does not depend on $\alpha, v_{0}$ and $f(t)$.

Note that it was established that a necessary condition for coercivity is the strong positivity of the operator coefficients $A(t)$. This condition is also sufficient in the Hölder spaces $C_{0}^{\alpha, \alpha}(E)$ with weight $t^{\alpha}$.

We now study Cauchy problem (14) in the spaces $L_{p}(E)=L_{p}([0, T], E)(1 \leq$ $p<\infty)$ of all strongly measurable $E$-valued functions $v(t)$ on $[0, T]$ for which the norm

$$
\|v\|_{L_{p}(E)}=\left(\int_{0}^{T}\|v(t)\|_{E}^{p} d t\right)^{\frac{1}{p}}
$$

is finite.

A function $v(t)$ is said to be absolutely continuous, if it has a derivative $v^{\prime}(t)$ for almost every $t$ such that $v^{\prime}(t) \in L_{1}(E)$, and if the Newton-Leibniz formula

$$
v(t)-v(\tau)=\int_{\tau}^{t} v^{\prime}(s) d s
$$

holds for all $t, \tau \in[0, T]$. Here, the integral is understood in the sense of Bochner.

A function $v(t)$ is said to be a solution of problem (14) in $L_{p}(E)$, if it is absolutely continuous, the functions $v^{\prime}(t)$ and $A(t) v(t)$ belong to $L_{p}(E)$, equation (14) is satisfied for almost every $t$, and $v(0)=v_{0}$. From this definition it follows that a necessary condition for the solvability of problem (14) in $L_{p}(E)$ is that $f(t)$ $\in L_{p}(E)$. It will be shown that in certain cases this condition is also sufficient for the solvability of problem (14). As concerns the boundary elements, in contrast to the situation considered earlier, from the solvability of problem (14) in $L_{p}(E)$ it follows only that $v_{0} \in E$. In the case of an unbounded operator $A(t)$ this does not allow us to prove the solvability of problem (14).

From the unique solvability of (14) it follows that the operator $v\left(t ; f(t), v_{0}\right)$ is bounded in $L_{p}(E)$ and one has the coercive inequality

$$
\left\|v^{\prime}\right\|_{L_{p}(E)}+\|A(.) v(t)\|_{L_{p}(E)} \leq M_{C}\left[\|f\|_{L_{p}(E)}+\left\|A(0) v_{0}\right\|_{E}\right],
$$


where $M_{C}\left(1 \leq M_{C}<\infty\right)$ does not depend on $v_{0}$ and $f(t)$.

In the case of Bochner spaces $L_{p}(E)$ with $p \in(1, \infty)$ the following extrapolation result was established for the first time by Sobolevskii in the paper [3].

Theorem 2.11. Suppose that the assumptions (16) and (17) hold and $0<\alpha \leq$ $\varepsilon<1$ Suppose problem (14) is well-posed in $L_{p_{0}}(E)$ for some $p_{0}, 1<p_{0}<\infty$. Then it is well-posed in $L_{p}(E)$ for any $p \in(1, \infty)$ and the following coercivity inequality holds:

$$
\begin{aligned}
& \left\|v^{\prime}\right\|_{L_{p}(E)}+\|A(.) v\|_{L_{p}(E)}+\left\|v^{\prime}\right\|_{C\left(E_{1-1 / p, p}\right)} \\
& \leq \frac{M\left(p_{0}\right) p^{2}}{p-1}\|f\|_{L_{p}(E)}+M\left\|A(0) v_{0}\right\|_{E_{1-1 / p, p}},
\end{aligned}
$$

where $M\left(p_{0}\right)$ and $M$ are independent of $p, v_{0}$ and $f(t)$.

Note that for the general space $E$ there are no coercivity inequalities in spaces $C(E)$ and $L_{1}(E)$. Furthermore, unconditional coercivity inequalities for the solutions of abstract differential equations of parabolic type were established in several Banach spaces by Sobolevskii in papers [4-6]. Note that for the first time Sobolevskii in the paper [5] successfully applied the theory of interpolation of linear positive operators to partial differential equations for obtaining new coercivity inequalities for the solutions of abstract parabolic equations. The application of these results to parabolic partial differential equations allowed one to obtain a series of new coercivity inequalities involving different norms with respect to $t$ and the space variables. His approach is different from application of classical techniques of PDEs. The main novelty of Sobolevskii's work is that he has successfully investigated the theory of partial differential equations independently from classical approaches (such as the maximum principle or others). One of the important aspects of Sobolevskii's work is that he has at the same time investigated theory of difference schemes for the approximate solutions of partial differential equations and theory of these partial differential equations. This approach was successful for the theory of partial differential equations and theory of their difference schemes. Moreover, Sobolevskii was really a perfect teacher and he was happy to give idea for students and investigate problems jointly with his colleagues and students. Therefore, Sobolevskii's many important results were established jointly with his students.

\subsection{Method of positive operators in investigation of difference schemes for parabolic equations}

The research interests of Sobolevskii in theory of difference schemes are broad and include stability and well-posedness of difference problems, construction and 
investigation of high order of accuracy difference schemes for partial differential equations, study of positivity of difference operators, and structure of fractional spaces generated by positive difference operators. The important results of Sobolevskii in the theory of difference schemes for parabolic equations were published in papers [7-36].

The central problem in the theory of difference schemes is the study of their stability. To prove stability of difference schemes, in a number of works, difference schemes were treated as operator equations in a Hilbert space, and the investigation was based on the symmetry properties of operator coefficient. This has led to $L_{2}$-stability estimates. Of great interest is the study of stability in the $C$-norm. In a number of papers the corresponding estimates were obtained for the simplest difference schemes approximating the Cauchy problem for parabolic equations and systems with constant coefficients and one space variable. The proof of stability is based on estimates of parabolic difference Green function (fundamental solutions). In the case of parabolic equations with variable coefficients and more than one space variable, stability in the $C$-norm is known to hold only for difference schemes that satisfy a maximum principle.

An important type of stability is the coercive stability (well-posedness) of difference schemes. There are no coercive estimates in the $C$-norm, because they do not hold for the corresponding differential equations. In a number of papers the coercive stability of difference schemes in the $L_{2}-$ and $L_{p}$ - norms were established.

Sobolevskii in the paper [30], treated for the first time difference schemes as operator equations in Banach spaces. In a Banach space, there is no notion of symmetry and for this reason the study of stability relies on the positivity property of the operator coefficients, expressed in terms of properties of their resolvents. Enlisting the theory of positive operators turned out to be effective in the investigation of such difference schemes (see [25-28] ). The coercivity inequality in the difference analogous of the Bochner spaces $L_{p}(\tau, E)$ holds for any $p \in(1, \infty)$ whenever it holds for at least one $p_{0} \in(1, \infty)$, i.e., here we have a result analogous to that in the differential case. Sobolevskii in the papers [14-16] successfully applied theory of interpolation of linear positive operators to theory of difference schemes for getting new coercivity inequalities in the spaces $L_{q}\left(\tau, E_{\alpha, q}^{\prime}\right)$ $(0<\alpha<1,1 \leq q \leq \infty)$. The application of these results to difference schemes for parabolic partial differential equations allowed one to obtain a series of new stability and coercivity inequalities involving different norms with respect to $t$ and the space variables. Note that these stability and coercivity inequalities of Crank-Nicolson difference scheme for parabolic partial differential equations in Hölder spaces which were established by Sobolevskii are new for one dimensional parabolic equations even (see [11-13, 15,31,33]). 
Moreover, this Sobolevskii's operator approach was very important in the study of the solution of the various problems for delay partial differential equations. Actually, Sobolevskii [37] successfully applied theory of interpolation of linear positive operators for delay differential equations of parabolic type. Stability estimates for the solutions of the first and second order of accuracy difference schemes for the approximate solution of this initial value problem for delay differential equations of parabolic type were presented. Stability estimates in Hölder norms for the solutions of the initial value problem of the delay differential and difference equations of the parabolic type were also obtained.

The application of the approximants $R_{j, l}^{k}(\tau A)$ in the construction of difference schemes in the case of an unbounded strongly positive operator $A$ acting in an arbitrary Banach space $E$ has been systematically developed by several authors. Difference schemes were studied for a class of operators that generate not only analytic, but also strongly continuous semigroups. Most of the results of the stability and coercive stability in the Banach spaces of the single-step difference schemes of a high order of accuracy generated by $R_{j, l}^{k}(\tau A)$ for parabolic equations were considered in the book [21]. The application of these results to the initial value problem for parabolic equations allowed one to obtain a series of new coercivity inequalities in difference analogous of various norms with respect to $t$ and $x$ for the solutions of difference schemes of the high order of accuracy in time and space variables.

However, the theory of the high order of accuracy single-step difference schemes for parabolic differential equations with variable operator coefficients has received less attention than parabolic differential equations with coefficients independent of time. On the segment $[0, T]$, we consider a uniform grid space

$$
[0, T]_{\tau}=\left\{t_{k}=k \tau, k=0,1, \cdots, N, N \tau=T\right\}
$$

with step $\tau>0$. Here, $N$ is a fixed positive integer. From formula (18) it follows that

$$
\begin{gathered}
\frac{1}{\tau}\left(v\left(t_{k}\right)-v\left(t_{k-1}\right)\right)+\frac{1}{\tau}\left(I-u\left(t_{k}, t_{k-1}\right)\right) v\left(t_{k-1}\right) \\
=\int_{t_{k-1}}^{t_{k}} u\left(t_{k}, s\right) f(s) d s, 1 \leq k \leq N, v(0)=v_{0} .
\end{gathered}
$$

The last system is called the single-step exact difference scheme for the solution of initial value problem (14).

The application of the Padè approximants to the Cauchy problem for parabolic equations with variable operator coefficients $A(t)$ acting in an arbitrary Banach space $E$ relies on the approximate formulas for expressions 


$$
v\left(t_{k}, t_{k-1}\right) \text { and } \frac{1}{\tau} \int_{t_{k-1}}^{t_{k}} v\left(t_{k}, s\right) f(s) d s .
$$

Unfortunately, in general case of operators $A(t)$ the explicit formula for $v\left(t_{k}, t_{k-1}\right)$ does not exist. Therefore, in this case we cannot use approaches of the construction of Padè difference schemes for parabolic equations with coefficients independent of time.

The single-step difference schemes of a high order of accuracy generated by an exact difference scheme for parabolic equations with variable operator coefficients $A(t)$ acting in an arbitrary Banach space $E$ were presented by Sobolevskii for the first time in [20]. The schemes were based on the series of interesting properties concerning the semigroup $\exp \{-s A(t)\}(s \geq 0)$ and the fundamental solution $v(t, s)$ of problem (14). These properties and a series of interesting estimates for $R_{j, l}^{i}\left(\tau A\left(t_{k}\right)\right)$ and $\widetilde{u}(k, m)$ permit us to study the stability and the coercive stability of presented difference schemes. Furthermore, the high order of accuracy difference schemes generated by the Taylor's decomposition on the two points for the approximate solutions of the Cauchy problem for parabolic equations with variable operator coefficients $A(t)$ acting in an arbitrary Banach space $E$ were presented. The utilization of the Taylor's decomposition on the two points in the construction of the single step difference schemes of the high order of accuracy for the approximate solutions of problem (14) is based on the following theorem.

Theorem 2.12. Suppose the function $v(t)(0 \leq t \leq T)$ has a $(p+q+1)$-th continuous derivative and $t_{k-1}, t_{k} \in[0, T]_{\tau}$. Then, the following relation holds:

$$
\begin{gathered}
v\left(t_{k}\right)-v\left(t_{k-1}\right)+\sum_{j=1}^{p} \alpha_{j} v^{(j)}\left(t_{k}\right) \tau^{j}-\sum_{j=1}^{q} \beta_{j} v^{(j)}\left(t_{k-1}\right) \tau^{j} \\
=\frac{(-1)^{p}}{(p+q) !} \int_{t_{k-1}}^{t_{k}}\left(t_{k}-s\right)^{q}\left(s-t_{k-1}\right)^{p} v^{(p+q+1)}(s) d s,
\end{gathered}
$$

where

$$
\begin{cases}\alpha_{j}=\frac{(p+q-j) ! p !(-1)^{j}}{(p+q) ! j !(p-j) !} & \text { for any } j, 1 \leq j \leq p, \\ \beta_{j}=\frac{(p+q-j) ! q !}{(p+q) ! j !(q-j) !} & \text { for any } j, 1 \leq j \leq q .\end{cases}
$$

From (21) it is clear that for the approximate solution of problem (14) it is necessary to find $v_{\left(t_{k}\right)}^{(j)}$ for any $j, 1 \leq j \leq p$ and $v_{\left(t_{k-1}\right)}^{(j)}$ for any $j, 1 \leq j \leq q$. Using the equation

$$
v^{\prime}(t)=-A(t) v(t)+f(t)
$$


we obtain

$$
v^{(j)}(t)=A_{j}(t) v(t)+F_{j}(t)
$$

where

$$
\left\{\begin{array}{l}
A_{1}(t)=-A(t), F_{1}(t)=f(t), A_{j}(t)=A_{j-1}^{(\prime)}(t)-A_{j-1}(t) A(t), \\
F_{j}(t)=F_{j-1}^{(\prime)}(t)+A_{j-1}(t) f(t) \text { for any } j, 2 \leq j \leq p
\end{array}\right.
$$

Replacing the $v^{(j)}(t)$ in (21) by (23) and neglecting the last term, we obtain the single-step difference schemes of $(p+q)$ - order of accuracy for the approximate solution of the problem (21)

$$
\begin{gathered}
\frac{u_{k}-u_{k-1}}{\tau}+\sum_{j=1}^{p} \alpha_{j} A_{j}\left(t_{k}\right) \tau^{j-1} u_{k}-\sum_{j=1}^{q} \beta_{j} A_{j}\left(t_{k-1}\right) \tau^{j-1} u_{k-1}=\varphi_{k}^{p, q}, \\
\varphi_{k}^{p, q}=-\sum_{j=1}^{p} \alpha_{j} F_{j}\left(t_{k}\right) \tau^{j-1}+\sum_{j=1}^{q} \beta_{j} F_{j}\left(t_{k-1}\right) \tau^{j-1}, 1 \leq k \leq N, u_{0}=y_{0} .
\end{gathered}
$$

The well-posedness of these difference schemes in the various Banach spaces were studied. The stability and coercive stability estimates in Banach norms for the solutions of the high order of accuracy difference schemes of the mixed type boundary value problems for parabolic equations were obtained. The study of the high order of accuracy difference schemes in the case of strongly positive operators $A(t)$ allowed to establish not only the stability but also the coercive stability in difference analogous of Banach spaces. The application of these abstract results to the mixed type boundary value problems for parabolic equations relies on the following facts: the strong positivity of an elliptic difference operator $A_{h}^{t, x}$ in the Banach space $E_{h}$, the well-posedness of the resolvent equation of $A_{h}^{t, x}$ in $E_{h}$ or in $E_{\alpha, p}^{\prime}\left(E_{h}, A_{h}^{t, x}\right), 1 \leq p \leq \infty$, the structure of fractional spaces $E_{\alpha, p}^{\prime}\left(E_{h}, A_{h}^{t, x}\right)$.

The strong positivity in difference analogue of weighted Hölder for an elliptic difference operator $A_{h}^{t, x}$ of second order of accuracy that approximates an elliptic operator without mixed derivatives was established in [42]. The strong positivity of the simplest multidimensional second-order elliptic difference operator in the $L_{p, h}$ and $C_{h}$-norms was established in [7-9]. The most thorough study of the strong positivity in the $C_{h}$-and $C_{h}^{\beta}$-norms of a wide class of operators that approximate elliptic operators of arbitrary order in $R^{n}$ was carried out in papers [38-41].

Finally, let us comment on the structure of fractional spaces. It is established that the norms in the spaces $E_{\alpha, p}^{\prime}\left(L_{p}\left(R_{h}^{n}\right), A_{h}^{t, x}\right)$ and $W_{p}^{2 m \alpha}\left(R_{h}^{n}\right)$ are equivalent 
uniformly in $h$ for any $0<\alpha<1 / 2 m$ and $1 \leq p \leq \infty$. This fact corresponds to the following equality, known in interpolation theory

$$
E_{\alpha, \rho}^{\prime}\left(L_{p}\left(R^{n}\right), A_{h}^{t, x}\right)=W_{p}^{2 m \alpha}\left(R^{n}\right), 0<\alpha<1 / 2 m, 1 \leq p \leq \infty,
$$

which in turn follows from the equality $D\left(A^{t, x}\right)=W_{p}^{2 m \alpha}\left(R^{n}\right)$ for an $2 m$-th order elliptic operator $A^{t, x}$ in $L_{p}\left(R^{n}\right), 1 \leq p \leq \infty$, via the real interpolation method. The alternative method of Sobolevski's investigation is based on estimates of fundamental solution of the resolvent equation for the operator $A_{h}^{t, x}$, allows us to consider also the cases $p=1$ and $p=\infty$.

\subsection{Partial differential equations of the elliptic type}

Sobolevskii in his paper [42] successfully applied the theory of analytic semigroups to the boundary value problem for elliptic differential equation

$$
-v^{\prime \prime}(t)+A v(t)=f(t) \quad(0 \leq t \leq T), v(0)=v_{0}, v(T)=v_{T}
$$

in an arbitrary Banach space $E$ with the linear positive operator $A$.

A function $v(t)$ is called a solution of problem (25) if the following conditions are satisfied:

i. $v(t)$ is a twice continuously differentiable on the segment $[0, T]$. The derivative at the endpoints of the segment are understood as the appropriate unilateral derivatives.

ii. The element $v(t)$ belongs to $D(A)$ for all $t \in[0, T]$, and the function $A v(t)$ is continuous on the segment $[0, T]$.

iii. $v(t)$ satisfies the equation and boundary conditions (25).

A solution of problem (25) defined in this manner will from now on be referred to as a solution of problem (25) in the space $C(E)=C([0, T], E)$. If $v(t)$ is a solution in $C(E)$ of problem (25), then the data of the problem satisfy the following conditions:

a) $f(t)$ belongs to $C(E)$;

b) $v_{0}$ and $v_{T}$ belongs to $D(A)$.

We say that problem (25) is well-posed in $C(E)$, if the following two conditions are satisfied: 
1. For any $f \in C(E)$ and $v_{0}, v_{T} \in D(A)$ there exists the unique solution $v(t)=v\left(t ; f(t), v_{0}, v_{T}\right)$ in $C(E)$ of problem (25). This means that an additive and homogeneous operator $v\left(t ; f(t), v_{0}, v_{T}\right)$ is defined which acts from $C(E) \times D(A) \times D(A)$ to $C(E)$ and gives the solution of (25) in $C(E)$.

2. $v\left(t ; f(t), v_{0}, v_{T}\right)$, regarded as an operator from $C(E) \times D(A) \times D(A)$ to $C(E)$, is continuous. Here $C(E) \times D(A) \times D(A)$ is understood as the normed space of the triplets $\left(f(t), v_{0}, v_{T}\right), f(t) \in C(E)$ and $v_{0}, v_{T} \in D(A)$, equipped with the norm

$$
\left\|\left(f(t), v_{0}, v_{T}\right)\right\|_{C(E) \times D(A) \times D(A)}=\|f\|_{C(E)}+\left\|v_{0}\right\|_{D(A)}+\left\|v_{T}\right\|_{D(A)} .
$$

Following the result due to Banach in $C(E)$ [36, p. 198] and these properties yield the following coercive inequality

$$
\left\|v^{\prime \prime}\right\|_{C(E)}+\|A v\|_{C(E)} \leq M_{C}\left[\|f\|_{C(E)}+\left\|A v_{0}\right\|_{E}+\left\|A v_{T}\right\|_{E}\right],
$$

where $M_{C}\left(1 \leq M_{C}<\infty\right)$ does not depend on $v_{0}, v_{T}$ and $f(t)$. Inequality (26) permits us to investigate the spectral properties of operator $A$ generated by the well-posedness in $C(E)$ of problem (25). For any $u \in D(A)$ and $\lambda \geq 0$ we will put

$$
\psi=\lambda u+A u
$$

Then, clearly, the function $e^{i \sqrt{\lambda} t} u(i=\sqrt{-1})$ is a solution in $C(E)$ of equation

$$
-v^{\prime \prime}(t)+A v(t)=f(t)
$$

with $f(t)=e^{i \sqrt{\lambda} t} \psi$. Let $w(t)$ be a smooth function defined on $[0, T]$ by the formula

$$
w(t)=\left\{\begin{array}{l}
0, \text { for any } t \in\left[0, \frac{T}{8}\right] \cup\left[\frac{7 T}{8}, T\right] \\
a(t), \text { for any } t \in\left[\frac{T}{8}, \frac{T}{4}\right] \cup\left[\frac{3 T}{4}, \frac{7 T}{8}\right] \\
1, \text { for any } t \in\left[\frac{T}{4}, \frac{3 T}{4}\right]
\end{array}\right.
$$

where $a(t)$ is a smooth function and $|a(t)| \leq 1$. Then, the function $v(t)=w(t) e^{i \sqrt{\lambda} t} u$ is a solution in $C(E)$ of problem (25) with $v(0)=$ $v(T)=0$ and

$$
f(t)=\left\{\begin{array}{l}
0, \text { for any } t \in\left[0, \frac{T}{8}\right] \cup\left[\frac{7 T}{8}, T\right], \\
-\left(a(t) e^{i \sqrt{\lambda} t}\right)_{t t} u+a(t) e^{i \sqrt{\lambda} t} A u, \text { for any } t \in\left[\frac{T}{8}, \frac{T}{4}\right] \cup\left[\frac{3 T}{4}, \frac{7 T}{8}\right], \\
e^{i \sqrt{\lambda} t} \psi, \text { for any } t \in\left[\frac{T}{4}, \frac{3 T}{4}\right] .
\end{array}\right.
$$

Therefore, from the coercive inequality (26) it follows that

$$
\lambda\|u\|_{E}+\|A u\|_{E}=\max _{t \in\left[\frac{T}{4}, \frac{3 T}{4}\right]}\left\|v^{\prime \prime}(t)\right\|_{E}+\max _{t \in\left[\frac{T}{4}, \frac{3 T}{4}\right]}\|A v(t)\|_{E}
$$




$$
\begin{gathered}
\leq \max _{t \in\left[\frac{T}{8}, \frac{7 T}{8}\right]}\left\|v^{\prime \prime}(t)\right\|_{E}+\max _{t \in\left[\frac{T}{8}, \frac{7 T}{8}\right]}\|A v(t)\|_{E} \\
=\left\|v^{\prime \prime}\right\|_{C(E)}+\|A v\|_{C(E)} \leq M_{C}\|f\|_{C(E)} \\
=M_{C} \max \left\{\|\psi\|_{E}, \max _{t \in\left[\frac{T}{8}, \frac{T}{4}\right] \cup\left[\frac{3 T}{4}, \frac{7 T}{8}\right]}\left\|-\left(a(t) e^{i \sqrt{\lambda} t}\right)_{t t} u+a(t) e^{i \sqrt{\lambda} t} A u\right\|_{E}\right\} .
\end{gathered}
$$

Since

$$
\begin{aligned}
& -\left(a(t) e^{i \sqrt{\lambda} t}\right)_{t t} u+a(t) e^{i \sqrt{\lambda} t} A u \\
= & a(t) e^{i \sqrt{\lambda} t} \psi-2 a^{\prime}(t) i \sqrt{\lambda} e^{i \sqrt{\lambda} t} u-a^{\prime \prime}(t) e^{i \sqrt{\lambda} t} u,
\end{aligned}
$$

we have that

$$
\begin{aligned}
& \left\|-\left(a(t) e^{i \sqrt{\lambda} t}\right)_{t t} u+a(t) e^{i \sqrt{\lambda} t} A u\right\|_{E} \\
\leq & |a(t)|\|\psi\|_{E}+2\left|a^{\prime}(t)\left\|\frac{d}{d t}\left(e^{i \sqrt{\lambda} t}\right)\right\|\|u\|_{E}+\right| a^{\prime \prime}(t)\|\| u \|_{E} \\
\leq & \|\psi\|_{E}+2 M_{1} \sqrt{\lambda}\|u\|_{E}+M_{2}\|u\|_{E},
\end{aligned}
$$

where

$$
M_{1}=\max _{t \in\left[\frac{T}{8}, \frac{T}{4}\right] \cup\left[\frac{3 T}{4}, \frac{7 T}{8}\right]}\left|a^{\prime}(t)\right|, M_{2}=\max _{t \in\left[\frac{T}{8}, \frac{T}{4}\right] \cup\left[\frac{3 T}{4}, \frac{7 T}{8}\right]}\left|a^{\prime \prime}(t)\right| .
$$

Thus,

$$
\lambda\|u\|_{E}+\|A u\|_{E} \leq M_{C}\left[\|\psi\|_{E}+2 M_{1} \sqrt{\lambda}\|u\|_{E}+M_{2}\|u\|_{E}\right] .
$$

Using the inequality

$$
0<2 \sqrt{\lambda}<\varepsilon \lambda+\varepsilon^{-1}
$$

for $\varepsilon>0$, we obtain

$$
\left[\lambda\left(1-\varepsilon M_{1} M_{C}\right)-\left(\varepsilon^{-1} M_{1} M_{C}+M_{2} M_{C}\right)\right]\|u\|_{E}+\|A u\|_{E} \leq M_{C}\|\psi\|_{E} .
$$

Let $0<\varepsilon<\left(M_{1} M_{C}\right)^{-1}$,

$$
\delta=\min _{0<\varepsilon<\left(M_{1} M_{C}\right)^{-1}}\left(\varepsilon^{-1} M_{1} M_{C}+M_{2} M_{C}\right)\left(1-\varepsilon M_{1} M_{C}\right)^{-1}
$$

and $\lambda \geq \delta+\eta$ for some $\eta>0$. Then, the following inequality

$$
(\lambda+1)\|u\|_{E}+\|A u\|_{E} \leq M_{p}(\eta)\|\psi\|_{E}
$$

holds for some $0 \leq M_{p}(\eta)<\infty$. This means that there exists the inverse operator $(\lambda I+A)^{-1}$, which is defined on the range $R(\lambda I+A)$ of the operator $\lambda I+A$. We will prove that $R(\lambda I+A)=E$ for sufficiently large positive $\lambda$. 
Then, from that it follows that there exists the inverse operator $(\lambda I+A)^{-1}$ and the following inequality is valid

$$
(\lambda+1)\left\|(\lambda I+A)^{-1} \psi\right\|_{E}+\left\|A(\lambda I+A)^{-1} \psi\right\|_{E} \leq M_{p}(\eta)\|\psi\|_{E}
$$

for all $\lambda, \lambda \geq \delta+\eta$ and $\psi \epsilon E$. Now, we will prove that $R(\lambda I+A)=E$ for all positive $\lambda$. We consider boundary value problem (25). Making the substitution $t=$ $\alpha \tau$ for $\alpha>0$, we obtain the well-posed boundary value problem in $C\left(\left[0, \frac{T}{\alpha}\right], E\right)$ :

$$
-\frac{1}{\alpha^{2}} \widetilde{v}^{\prime \prime}(\tau)+A \widetilde{v}(\tau)=\widetilde{f}(\tau)\left(0 \leq \tau \leq \frac{T}{\alpha}\right), \widetilde{v}(0)=v_{0}, \widetilde{v}\left(\frac{T}{\alpha}\right)=v_{T} .
$$

Let $\tilde{v}(\tau)$ be the solution of this problem for $v_{0}=v_{T}=0$ and $\tilde{f}(\tau)=f_{0}$, where $f_{0}$ is an arbitrary element of $E$. Since $f_{0}$ and $A$ do not depend in $\tau$, we have that $\widetilde{v}(\tau)=\widetilde{v}\left(\frac{T}{2 \alpha}-\tau\right)$ for all $0 \leq \tau \leq \frac{T}{2 \alpha}$. Then, in particular, it follows that $\widetilde{v}^{\prime}(0)=-\widetilde{v}^{\prime}\left(\frac{T}{\alpha}\right)$. Finally, choosing $\sqrt{\lambda} \frac{T}{\alpha}=(2 m+1) \pi$ for some $m=1,2, \cdots$ and integrating by parts, we obtain

$$
\begin{gathered}
-\int_{0}^{\frac{T}{\alpha}} \widetilde{v}^{\prime \prime}(\tau) e^{i \sqrt{\lambda} \tau} d \tau=\left[-\widetilde{v}^{\prime}\left(\frac{T}{\alpha}\right) e^{i \sqrt{\lambda} \frac{T}{\alpha}}-\widetilde{v}^{\prime}(0)\right]+i \sqrt{\lambda}\left[\widetilde{v}\left(\frac{T}{\alpha}\right) e^{i \sqrt{\lambda} \frac{T}{\alpha}}-\widetilde{v}(0)\right] \\
-(i \sqrt{\lambda})^{2} \int_{0}^{\frac{T}{\alpha}} \widetilde{v}(\tau) e^{i \sqrt{\lambda} \tau} d \tau=\lambda \int_{0}^{\frac{T}{\alpha}} \widetilde{v}(\tau) e^{i \sqrt{\lambda} \tau} d \tau .
\end{gathered}
$$

Therefore,

$$
\begin{gathered}
-\int_{0}^{\frac{T}{\alpha}} \widetilde{v}^{\prime \prime}(\tau) e^{i \sqrt{\lambda} \tau} d \tau=\left[-\widetilde{v}^{\prime}\left(\frac{T}{\alpha}\right) e^{i \sqrt{\lambda} \frac{T}{\alpha}}-\widetilde{v}^{\prime}(0)\right]+i \sqrt{\lambda}\left[\widetilde{v}\left(\frac{T}{\alpha}\right) e^{i \sqrt{\lambda} \frac{T}{\alpha}}-\widetilde{v}(0)\right] \\
\frac{\lambda}{\alpha^{2}} \int_{0}^{\frac{T}{\alpha}} \widetilde{v}(\tau) e^{i \sqrt{\lambda} \tau} d \tau+A \int_{0}^{\frac{T}{\alpha}} \widetilde{v}(\tau) e^{i \sqrt{\lambda} \tau} d \tau=\int_{0}^{\frac{T}{\alpha}} f_{0} e^{i \sqrt{\lambda} \tau} d \tau=-\frac{2}{i \sqrt{\lambda}} f_{0}
\end{gathered}
$$

for all $\lambda, \lambda>0$ and $f_{0} \epsilon E$. This means that $R(\lambda I+A)=E$ for all $\sqrt{\lambda} \frac{T}{\alpha}=$ $(2 m+1) \pi$ and $m=1,2, \cdots$. From that it follows that there exists the bounded inverse $(\lambda I+A)^{-1}$ for all $\lambda \geq \delta+\eta$. An operator $A$ acting in a Banach space $E$ and having a dense domain $D(A)$ is called positive if the operator $\lambda I+A$ has bounded in $E$ inverse for all $\lambda, \lambda \geq 0$, and the estimate

$$
\left\|(\lambda I+A)^{-1}\right\|_{E \rightarrow E} \leq M_{p}(\lambda+1)^{-1}
$$

is satisfied for some $1 \leq M_{p}<\infty$. Note that, as seen in above computations, we are not able to obtain the estimate (27) for all $\lambda, \lambda \geq 0$. 
Is the positivity of the operator $A$ a sufficient condition for the well-posedness of (25) in $C(E)$ ? As it turns out, problem (25) is not well posed in $C(E)$ for all such positive operators.

Note that from estimate (27), it follows that the operator $\lambda I+A$ has bounded inverse for all complex numbers

$$
\lambda=\sigma+i \tau \in G_{\varepsilon}^{+}=G_{\varepsilon}^{+}\left(M_{p}\right)(0<\varepsilon<1),
$$

such that

$$
|\tau| \leq \frac{1-\varepsilon}{M_{p}}(1+\sigma), \text { for } \sigma \geq 0
$$

or

$$
\left(\sigma^{2}+\tau^{2}\right)^{1 / 2} \leq \frac{1-\varepsilon}{M_{p}}, \text { for } \sigma \leq 0
$$

and the estimate

$$
\left\|(\lambda I+A)^{-1}\right\|_{E \rightarrow E} \leq M_{1} \varepsilon^{-1}(1+|\lambda|)^{-1}
$$

holds for some $M_{1} \in[1, \infty)$ which is independent of $\varepsilon \in(0,1)$. It means that the spectrum $\sigma(A)$ of $A$ is the subset of set $G_{\varepsilon}^{-}=-G_{\varepsilon}^{+}$and inside of $G_{\varepsilon}^{-}$and on its boundary $\partial G_{\varepsilon}^{-}$the resolvent $(\lambda I-A)^{-1}$ is subject to the bound

$$
\left\|(\lambda I-A)^{-1}\right\|_{E \rightarrow E} \leq M_{1} \varepsilon^{-1}(1+|\lambda|)^{-1} .
$$

Let $\psi(z)$ be an analytic function on the neighborhood of $\sigma(A)$, and suppose that $\psi$ satisfies the estimate

$$
(1+|z|)^{\alpha}|\psi(z)| \leq M_{2}
$$

for some $0<\alpha<\infty, 1 \leq M_{2}<\infty$. Then the operator Cauchy-Riesz integral

$$
\psi(A)=\frac{1}{2 \pi i} \int_{\partial G_{\varepsilon}^{-}} \psi(z)(z I-A)^{-1} d z
$$

converges as a function of $A$ in the operator norm and defines a bounded linear operator $\psi(A)$. In particular, the function $\psi(A)=z^{-\alpha}$ defines a bounded operator $A^{-\alpha}$ whenever $\alpha>0$. The positive powers $A^{\alpha}=\left(A^{-\alpha}\right)^{-1}(\alpha>0)$ of the operator $A$ are defined and unbounded, and their domains $D\left(A^{a}\right)$ are dense in $E$ if $A$ is unbounded. One has the continuous embedding $D\left(A^{\alpha}\right) \subset D\left(A^{\beta}\right)$ if $\beta<\alpha$. The following moment inequality holds:

$$
\begin{aligned}
\left\|A^{\alpha} u\right\|_{E} & \leq M(\alpha, \beta)\left\|A^{\beta} u\right\|_{E}^{\alpha / \beta}\|u\|_{E}^{1-\alpha / \beta}, \\
0 & \leq \alpha \leq \beta<\infty, u \in D\left(A^{\beta}\right)
\end{aligned}
$$


for some $M(\alpha, \beta) \in[1, \infty)$ that does not depend on $u \in D\left(A^{\beta}\right)$. The operator $A^{1 / 2}$ has nicer spectral properties than the positive operator $A$. Indeed, using the identity

$$
\lambda I+A=\left(\sqrt{\lambda} I-A^{\frac{1}{2}}\right)\left(\sqrt{\lambda} I+A^{\frac{1}{2}}\right),
$$

the inequality (30) for $\alpha=\frac{1}{2}, \beta=1$ and the estimate (28) we obtain

$$
\begin{gathered}
\left\|\left(\sqrt{\lambda} I-A^{\frac{1}{2}}\right)^{-1}\right\|_{E \rightarrow E}=\left\|\left(\sqrt{\lambda} I+A^{\frac{1}{2}}\right)(\lambda I+A)^{-1}\right\|_{E \rightarrow E} \\
\leq \sqrt{|\lambda|}\left\|(\lambda I+A)^{-1}\right\|_{E \rightarrow E}+M(\alpha, \beta)\left\|A(\lambda I+A)^{-1}\right\|_{E \rightarrow E}^{\frac{1}{2}}\left\|(\lambda I+A)^{-1}\right\|_{E \rightarrow E}^{\frac{1}{2}} \\
\leq \sqrt{|\lambda|} M_{1} \varepsilon^{-1}(1+|\lambda|)^{-1} \\
+M(\alpha, \beta)\left(1+M_{1} \varepsilon^{-1}|\lambda|(1+|\lambda|)^{-1}\right)\left(M_{1} \varepsilon^{-1}(1+|\lambda|)^{-1}\right)^{\frac{1}{2}} .
\end{gathered}
$$

Thus, the operator $\sqrt{\lambda} I-A^{\frac{1}{2}}$ has a bounded inverse for $\lambda \in G_{\varepsilon}^{-}$, and the estimate

$$
\left\|\left(\sqrt{\lambda} I-A^{\frac{1}{2}}\right)^{-1}\right\|_{E \rightarrow E} \leq M_{3} \varepsilon^{-1}(1+\sqrt{|\lambda|})^{-1}
$$

holds for some $M_{3} \in[1, \infty)$ does not depend on $\epsilon$ and $\lambda$. This means that $B=A^{\frac{1}{2}}$ is a strongly positive operator in the Banach space $E$. Therefore, the operator $-B$ is a generator of an analytic semigroup $\exp \{-t B\}(t \geq 0)$ with exponentially decreasing norm, when $t \longrightarrow \infty$, i. e., the following estimates

$$
\|\exp (-t B)\|_{E \rightarrow E},\|t B \exp (-t B)\|_{E \rightarrow E} \leq M(B) e^{-\alpha(B) t}(t>0)
$$

hold for some $M(B) \in[1, \infty), a(B) \in(0, \infty)$. From that it follows that the operator $I-e^{-2 T B}$ has the bounded inverse and the following estimate holds:

$$
\left\|\left(I-e^{-2 T B}\right)^{-1}\right\|_{E \rightarrow E} \leq M(B)\left(1-e^{-2 T \alpha(B)}\right)^{-1} .
$$

It is easy to show that $v(t)$ defined on $[0, T]$ by the formula

$$
\begin{gathered}
v(t)=\left(I-e^{-2 T B}\right)^{-1}\left\{\left(e^{-t B}-e^{-(2 T-t) B}\right) v_{0}+\left(e^{-(T-t) B}-e^{-(T+t) B}\right) v_{T}\right. \\
\left.-\left(e^{-(T-t) B}-e^{-(T+t) B}\right)(2 B)^{-1} \int_{0}^{T}\left(e^{-(T-s) B}-e^{-(T+s) B}\right) f(s) d s\right\} \\
+(2 B)^{-1} \int_{0}^{T}\left(e^{-|t-s| B}-e^{-(t+s) B}\right) f(s) d s
\end{gathered}
$$

is the unique solution in $C(E)$ of problem (25) if, for example, $v_{0}, v_{T} \in D\left(A^{2}\right)$ and $A f(t) \in C(E)$ or $f^{\prime}(t) \in C(E)$. Sufficient conditions for the well-posedness 
of boundary value problem (25) can be established, if one considers this problem in certain spaces $F(E)$ of smooth $E$-valued functions on $[0, T]$.

A function $v(t)$ is said to be a solution of problem (25) in $F(E)$, if it is a solution of this problem in $C(E)$ and the function $v^{\prime \prime}(t)$ and $A v(t)$ belong to $F(E)$.

As in the case of the space $C(E)$, we say that problem (25) is well-posed in $F(E)$ if the following two conditions are satisfied:

1. For any $f \in F(E)$ and $v_{0}, v_{T} \in D(A)$, there exists a unique solution $v(t)=$ $v\left(t ; f(t), v_{0}, v_{T}\right)$ in $F(E)$ of problem (25). This means that an additive and homogeneous operator $v\left(t ; f(t), v_{0}, v_{T}\right)$ is defined which acts from $F(E) \times$ $D(A) \times D(A)$ to $F(E)$ and gives the solution of (25) in $F(E)$.

2. $v\left(t ; f(t), v_{0}, v_{T}\right)$, regarded as an operator from $F(E) \times D(A) \times D(A)$ to $F(E)$, is continuous. Here, $F(E) \times D(A) \times D(A)$ is understood as the normed space of the triplets $\left(f(t), v_{0}, v_{T}\right), f(t) \in F(E)$ and $v_{0}, v_{T} \in D(A)$, equipped with the norm

$$
\left\|\left(f(t), v_{0}, v_{T}\right)\right\|_{F(E) \times D(A) \times D(A)}=\|f\|_{F(E)}+\left\|v_{0}\right\|_{D(A)}+\left\|v_{T}\right\|_{D(A)} .
$$

First, we set $F(E)$ is equal to $C_{0 T}^{\alpha, \alpha}(E)=C_{0 T}^{\alpha, \alpha}([0, T], E), 0<\alpha<1$, the Banach space obtained by completion of the set of smooth $E$-valued functions $\varphi(t)$ on $[0, T]$ in the norm

$$
\|\varphi\|_{C_{0 T}^{\alpha, \alpha}(E)}=\|\varphi\|_{C(E)}+\sup _{0 \leq t<t+\tau \leq T} \frac{\|\varphi(t+\tau)-\varphi(t)\|_{E}}{\tau^{\alpha}}(T-t)^{\alpha}(t+\tau)^{\alpha} .
$$

As in the case of the space $C(E)$, from the well-posedness of boundary value problem (25) one derives the coercivity inequality

$$
\left\|v^{\prime \prime}\right\|_{C_{0 T}^{\alpha, \alpha}(E)}+\|A v\|_{C_{0 T}^{\alpha, \alpha}(E)} \leq M_{C}(\alpha)\left[\|f\|_{C_{0 T}^{\alpha, \alpha}(E)}+\left\|A v_{0}\right\|_{E}+\left\|A v_{T}\right\|_{E}\right],
$$

where $M_{C}(\alpha)\left(1 \leq M_{C}(\alpha)<\infty\right)$ does not depend on $v_{0}, v_{T}$ and $f(t)$.

Theorem 2.13. Suppose $v_{0}, v_{T} \in D(A), f(t) \in C_{0 T}^{\alpha, \alpha}(E)(0<\alpha<1)$. Then, the boundary value problem (25) is well-posed in Hölder space $C_{0 T}^{\alpha, \alpha}(E)$, if $A$ is a positive operator in Banach space E. For the solution $v(t)$ in $C_{0 T}^{\alpha, \alpha}(E)$ of the boundary value problem, the coercive inequality $\left\|v^{\prime \prime}\right\|_{C_{0 T}^{\alpha, \alpha}(E)}+\|A v\|_{C_{0 T}^{\alpha, \alpha}(E)} \leq \frac{M}{\alpha(1-\alpha)}\|f\|_{C_{0 T}^{\alpha, \alpha}(E)}+M\left[\left\|A v_{0}\right\|_{E}+\left\|A v_{T}\right\|_{E}\right]$ holds, where $M$ is dependent of $\alpha, v_{0}, v_{T}$ and $f(t)$. 
The research interests of Sobolevskii in theory of difference schemes are broad and include stability and well-posedness of difference problems, construction and investigation of high order of accuracy difference schemes for elliptic differential equations. The important results of Sobolevskii in theory of difference schemes for elliptic equations were published in papers [42-54].

\subsection{Partial differential equations of the hyperbolic type}

Sobolevskii in the paper [55] successfully applied for the first time the theory of positive operators to the abstract Cauchy problem for hyperbolic equations

$$
v^{\prime \prime}(t)+A v(t)=f(t) \quad(0 \leq t \leq T), v(0)=v_{0}, v^{\prime}(0)=v_{0}^{\prime}
$$

where $A$ is a self -adjoint positive definite operator on a Hilbert space $H$.

A function $v(t)$ is called a solution of problem (35) if the following conditions are satisfied:

i. $v(t)$ is twice continuously differentiable on the segment $[0, T]$. The derivatives at the endpoints of the segment are understood as the appropriate unilateral derivatives.

ii. The element $v(t)$ belongs to $D(A)$ for all $t \in[0, T]$ and the function $A v(t)$ is continuous on the segment $[0, T]$.

iii. $v(t)$ satisfies the equations and initial conditions (35).

Theorem 2.14. Suppose that $v_{0} \in D(A), v_{0}^{\prime} \in D\left(A^{\frac{1}{2}}\right)$ and $f(t)$ are continuously differentiable on $[0, T]$ function. Then, there is a unique solution of problem (35) and the following stability inequalities

$$
\begin{gathered}
\max _{0 \leq t \leq T}\|v(t)\|_{H} \leq M\left[\left\|v_{0}\right\|_{H}+\left\|A^{-1 / 2} v_{0}^{\prime}\right\|_{H}+\max _{0 \leq t \leq T}\left\|A^{-1 / 2} f(t)\right\|_{H}\right], \\
\max _{0 \leq t \leq T}\left\|A^{1 / 2} v(t)\right\|_{H} \leq M\left[\left\|A^{1 / 2} v_{0}\right\|_{H}+\left\|v_{0}^{\prime}\right\|_{H}+\max _{0 \leq t \leq T}\|f(t)\|_{H}\right], \\
\max _{0 \leq t \leq T}\left\|\frac{d^{2} v(t)}{d t^{2}}\right\|_{H}+\max _{0 \leq t \leq T}\|A v(t)\|_{H} \leq M\left[\left\|A v_{0 H}+\right\| A^{1 / 2} v_{0}^{\prime} \|_{H}\right. \\
\left.+\|f(0)\|_{H}+\int_{0}^{T}\left\|f^{\prime}(t)\right\|_{H} d t\right]
\end{gathered}
$$

hold, where $M$ does not depend on $f(t), t \in[0, T]$ and $v_{0}, v_{0}^{\prime}$. 
The method of operators as a tool for the investigation of the solution to hyperbolic differential equations in Hilbert and Banach spaces has been systematically developed by several authors. A large cycle of these works on stability of difference schemes for hyperbolic partial differential equations was established under the assumption that the magnitude of the grid steps $\tau$ and $h$ with respect to the time and space variables are connected. In abstract terms this means, in particular, that the condition $\tau\left\|A_{h}\right\| \rightarrow 0$ when $\tau \rightarrow 0$ is satisfied.

Of great interest is the study of absolute stable difference schemes of a high order of accuracy for hyperbolic partial differential equations, in which stability was established without any assumptions in respect of the grid steps $\tau$ and $h$. Sobolevskii in the paper [56] the first order of accuracy absolute stable difference scheme

$$
\left\{\begin{array}{l}
\tau^{-2}\left(u_{k+1}-2 u_{k}+u_{k-1}\right)+A u_{k+1}=f_{k}, \\
f_{k}=f\left(t_{k}\right), t_{k}=k \tau, 1 \leq k \leq N-1, N \tau=T, \\
\tau^{-1}\left(u_{1}-u_{0}\right)+i A^{1 / 2} u_{1}=i A^{1 / 2} u_{0}+\psi, u_{0}=\varphi
\end{array}\right.
$$

for the solutions of problem (35) was studied. The following stability inequalities for the solutions of difference scheme (36) were established.

Theorem 2.15. Let $\varphi \in D(A), \psi \in D\left(A^{1 / 2}\right)$. Then, for the solution of difference scheme (36) the stability inequalities

$$
\begin{gathered}
\left\|u_{k}\right\|_{H} \leq \sum_{s=1}^{k-1}\left\|A^{-1 / 2} f_{s}\right\|_{H} \tau+\left\|A^{-1 / 2} \psi\right\|_{H}+\|\varphi\|_{H}, 1 \leq k \leq N, \\
\left\|A^{1 / 2} u_{k}\right\|_{H} \leq \sum_{s=1}^{k-1}\left\|f_{s}\right\|_{H} \tau+\left\|A^{1 / 2} \varphi\right\|_{H}+\|\psi\|_{H}, 1 \leq k \leq N, \\
\left\|A u_{k} \quad\right\| \quad H \leq 2 \sum_{s=2}^{k-1}\left\|f_{s}-f_{s-1}\right\|_{H} \\
+\left\|f_{1}\right\|_{H}+\left\|A^{1 / 2} \psi\right\|_{H}+\|A \varphi\|_{H}, 1 \leq k \leq N
\end{gathered}
$$

hold.

The application of these results to hyperbolic partial differential equations allows one to obtain a series of stability inequalities. His approach is different from application of classical technics of PDE. The main aspect of Sobolevskii's work is that he has successfully investigated the theory of partial differential equations independently from classical approaches. 
However, for practical realization of these difference schemes it is first necessary to construct an operator $A^{1 / 2}$. This consideration is very difficult even with the aid of computers. Therefore, in spite of theoretical results the role of their application of a numerical solution for an initial value problem is not great. The new first and second order of accuracy difference schemes approximately solving the abstract initial value problem for hyperbolic equations in a Hilbert spaces were presented in [56]. Applying this operator approach the stability estimates for the solution of these difference schemes were obtained.

Moreover, in [58] the high order of accuracy two-step difference schemes generated by an exact difference scheme for the numerical solutions of this problem were presented. The convergence and stability estimates for the solutions of these difference schemes were established. Sobolevskii's important results in theory of difference schemes for hyperbolic were published in papers [55-61].

These and other fundamental scientific results of Sobolevskii have been included in the treasury of the world of mathematical science!

\section{Selected books authored or co-authored by Sobolevskii}

1. M. A. Krasnosel'skii, P. P. Zabreiko, E. I. Pustyl'nik and P. E. Sobolevskii, Integral'nye operatory $v$ prostranstvah summiruemyh funkcii (Integral Operators in Spaces of Integrable Functions), Nauka, Moscow, 1966, 499 pages.

The investigation of many mathematical problems is significantly simplified, if it is possible to reduce them to equations involving continuous or completely continuous operators in function spaces. In particular, this is true for nonlinear boundary value problems and for integro-differential and integral equations. To effect a transformation to equations with continuous or completely continuous operators, it is usually necessary to reduce the original problem to one involving integral equations. Here, negative and fractional powers of those unbounded differential operators which constitute 'principal parts' of the original problem, are used in an essential way. Next, there is chosen or constructed a function space in which the corresponding integral operator possesses sufficiently good properties. Once such a space is found, the original problem can often be analyzed by applying general theorems (Fredholm theorems in the study of linear equations, fixed point principles in the study of nonlinear equations, methods of the theory of cones in the study of positive solutions, etc.). In other words, the investigation of many problems is effectively divided into three independent parts: transformation to an integral equation, investigation of the corresponding integral expression as an operator acting in function spaces, and, finally, application of general methods of functional analysis to the investigation of the linear and nonlinear equations. 


\section{A. Ashyralyev and P. E. Sobolevskii, Well-Posedness of Parabolic Difference} Equations, Vol. 69 of Operator Theory: Advances and Applications, Birkhäuser Verlag, Basel, Boston, Berlin, 1994, 349 pages.

Mathematical models described by various real dynamic processes can be reduced to boundary value problems for partial differential equations. A well-known and widely applied method of approximating the solutions of various problems of partial differential equations is the method of difference schemes. The main theoretical questions center around their accuracy and stability. The advancements in modern computer technology make it possible to solve difficult and computationally demanding problems. Hence, a task of current interest of this monograph is the construction and investigation of highly accurate difference schemes for various boundary value problems for partial differential equations. The monograph is devoted to the construction and investigation of highly accurate difference schemes for parabolic boundary value problems, based on Padè approximations. The investigation is based on a new notion of positivity of difference operators in Banach spaces, which allows one to deal with difference schemes of arbitrary order of accuracy. Establishing coercivity inequalities allows one to obtain sharp convergence rates, that is two-sided estimates. The proofs are based on results from interpolation theory of linear operators. This approach has led to the development of the theory of difference schemes of high order accuracy for various boundary value problems for partial differential equations.

3. A. Ashyralyev and P. E. Sobolevskii, New Difference Schemes for Partial Differential Equations, Birkhäuser Verlag: Basel. Boston. Berlin, Vol.148 of Operator Theory: Advances and Applications, 2004, 443 pages.

The monograph is devoted to the construction and investigation of the new high order of accuracy difference schemes for approximating the solutions of regular and singular perturbation boundary value problems for partial differential equations. The construction is based on the exact difference scheme and Taylor's decomposition on the two or three points. This approach permits essentially to extend and enlarge the class of problems where the theory of difference methods is applicable. Namely, now it is possible to investigate the differential equations with variable coefficients and regular and singular perturbation boundary value problems. The investigation is based on new coercivity inequalities.

At present, many researchers apply this methodology to investigate various problems of mathematical physics and stochastic models which appear in applied problems.

4. P. E. Sobolevskii, Difference Methods for the Approximate Solution of Partial Differential Equations, Izdat. Voronezh. Gosud. Univ., Voronezh, 1975, 150 pages (in Russian). 


\section{Bibliography}

[1] P. E. Sobolevskii, On equations with operators forming an acute angle, Dokl. Akad. Nauk SSSR 116(5) (1957), 754-757 (in Russian).

[2] P. E. Sobolevskii, M. F. Tiunchik, The angle between parabolic operators, in: Trudy Nauchn. -Issled. Inst. Mat. Voronezh. Gos. Univ. No. 5(1971), 103-113 (in Russian).

[3] P. E. Sobolevskii, Coerciveness inequalities for abstract parabolic equations, Dokl. Akad. Nauk SSSR 197(1) (1964), 52-55 (in Russian).

[4] V. P. Anosov and P. E. Sobolevskii, The coercive solvability of parabolic equations, Mat. Zametki 11(2) (1972), 409-419 (in Russian).

[5] P. E. Sobolevskii, Some properties of the solutions of differential equations in fractional spaces, in: Trudy Nauchn.-Issled. Inst. Mat. Voronezh. Gos. Univ. No. 74(1975), 68-76 (in Russian).

[6] A. Ashyralyev, A. Hanalyev and P. E. Sobolevskii, Coercive solvability of nonlocal boundary value problem for parabolic equations, Abstr. Appl. Anal. 6(1) (2001), 5361.

[7] Kh. Alibekov and P. E. Sobolevskii, Stability of difference schemes for parabolic equations, Dokl. Acad. Nauk SSSR 232(4) (1977), 737-740 (in Russian).

[8] Kh. Alibekov and P. E. Sobolevskii, Stability and convergence of difference schemes of the high order for parabolic differential equations, Ukrain. Mat. Zh. 31(6) (1979), 627-634 (in Russian).

[9] Kh. Alibekov and P. E. Sobolevskii, Stability and convergence of difference schemes of the high order for parabolic partial differential equations, Ukrain. Mat. Zh. 32(3) (1980), 291-300 (in Russian).

[10] A. Ashyralyev, I. Karatay and P. E. Sobolevskii, On well-posedness of the nonlocal boundary value problem for parabolic difference equations, Discrete Dyn. Nat. Soc., 2004(2) (2004), 273-286.

[11] A. Ashyralyev and P. E. Sobolevskii, Coercive stability of a Crank-Nicolson difference scheme in $\widetilde{C}_{0}^{\alpha}$ spaces, in: Approximate Methods for Investigations of Differential Equations and their Applications, Kuybishev (1982), 16-24 (in Russian).

[12] A. Ashyralyev and P. E. Sobolevskii, Correct solvability of a Crank-Nicolson difference scheme for parabolic equations, Izv. Akad. Nauk Turkmen. SSR Ser. Fiz. -Tekhn. Khim. Geol. Nauk 6 (1981), 10-16 (in Russian).

[13] A. Ashyralyev and P. E. Sobolevskii, The Crank-Nicolson difference scheme for differential equations in a Banach space with a time- dependent operator, Izv. Akad. Nauk Turkmen. SSR Ser. Fiz. -Tekhn. Khim. Geol. Nauk 3 (1982), 3-9 (in Russian).

[14] A. Ashyralyev and P. E. Sobolevskii, On the coercive stability of the difference schemes for abstract parabolic equations with variable operator coefficients in interpolation spaces, in: Investigations on the Theory of Differential Equations, Ashgabat (1983), 65-77 (in Russian). 
[15] A. Ashyralyev and P. E. Sobolevskii, The theory of interpolation of linear operators and the stability of difference schemes, Dokl. Akad. Nauk SSSR 275(6) (1984), 12891291 (in Russian).

[16] A. Ashyralyev and P. E. Sobolevskii, Stability of difference schemes for parabolic equations in interpolation spaces, in: Applied Methods of Functional Analysis, Voronezh(1985), 9-17 (in Russian).

[17] A. Ashyralyev and P. E. Sobolevskii, Coercive stability of difference schemes of first and second order of approximation for parabolic equations with coefficients independent of time, Izv. Akad. Nauk Turkmen. SSR Ser. Fiz. -Tekhn. Khim. Geol. Nauk 6 (1985), 3-11 (in Russian).

[18] A. Ashyralyev and P. E. Sobolevskii, Stability in a Holder norms of difference schemes for parabolic equations, in: Numerical Methods for the Solving of the Transport Equations, Tartu(1986), 20-23 (in Russian).

[19] A. Ashyralyev and P. E. Sobolevskii, Coercive stability of a multidimensional difference elliptic equations of $2 \mathrm{~m}$-th order with variable coefficients, in: Investigations in the Theory of Differential Equations, Ashgabat(1987), 31-43 (in Russian).

[20] A. Ashyralyev and P. E. Sobolevskii, Difference schemes of the high order of accuracy for parabolic equations with variable coefficients, Dokl. Akad. Nauk Ukrainian SSR, Ser. A Fiz. -Mat. and Tech. Sciences 6 (1988), 3-7 (in Russian).

[21] A. Ashyralyev and P. E. Sobolevskii, Well-Posedness of Parabolic Difference Equations, Vol. 69 of Operator Theory: Advances and Applications, Birkhäuser Verlag: Basel, Boston, Berlin , 1994.

[22] A. Ashyralyev and P. E. Sobolevskii, Well-posed solvability of the Cauchy problem for the difference equations of the parabolic type, Nonlinear Analysis. Theory. Methods and Applications 24(2) (1995), 257-264.

[23] A. Ashyralyev and P. E. Sobolevskii, Theory of positive operators and stability of the difference schemes, in: Some Problems of Applied Mathematics, Fatih University, Istanbul (2000), 61-69.

[24] A. Ashyralyev, Y. Sozen and P. E. Sobolevskii, A note on the parabolic differential and difference equations, Abstr. Appl. Anal. 2007 (2007), Article ID: 61659, 16 pages.

[25] A. E. Polichka and P. E. Sobolevskii, New $L_{p}$-estimates for parabolic difference problems, Zh. Vychisl. Mat. i Rat. Fiz. 17(5) (1976), 1155-1163 (in Russian).

[26] A. E. Polichka and P. E. Sobolevskii, Rothe's method of approximate solution of the Cauchy problem for differential equations in a Bochner space with a variable unbounded operator, Differentsial' nye Uravneniya 12(9) (1976), 1593-1704 (in Russian).

[27] A. E. Polichka and P. E. Sobolevskii, Correct solvability of a difference boundary value problem in Bochner space, Ukrain. Mat. Zh. 28(4) (1976), 711-523 (in Russian). 
[28] A. E. Polichka and P. E. Sobolevskii, Correct solvability of parabolic difference equations in Bochner spaces, Trudy Bosk. Mat. Obshch. 36 (1978), 29-57 (in Russian).

[29] A. E. Polichka and P. E. Sobolevskii, Some properties of the Crank-Nicolson scheme, in: Computations with Sparse Matrices, pp. 115-122, Novosibirsk, 1976 (in Russian).

[30] P. E. Sobolevskii, The coercive solvability of difference equations, Dokl. Akad. Nauk SSSR 201 (1971), 1063-1046 (in Russian).

[31] P. E. Sobolevskii, On the stability and convergence of the Crank-Nicolson scheme, in:Variational-Difference Methods in Mathematical Physics, pp. 146-151, Vychisl. Tsentr Sibirsk. Otdel. Akad. Nauk SSSR, Novosibirsk, 1974 (in Russian).

[32] P. E. Sobolevskii, The theory of semigroups and the stability of difference schemes, in:Operator Theory in Function Spaces (Proc. School, Novosibirsk , 1975), pp. 304337, "Nauka”, Sibirsk. Otdel. Akad. Nauk SSSR, Novosibirsk, 1977 (in Russian).

[33] P. E. Sobolevskii, On the Crank-Nicolson difference scheme for parabolic equations, in: Nonlinear Oscillations and Control Theory, pp. 98-106, Izhevsk, 1978 (in Russian).

[34] P. E. Sobolevskii and Hoang Van Lai, Algorithms of optimal type for the approximate solution of parabolic equations, in:Vychisl. Systemy no. 72(1977), 79-91 (in Russian).

[35] P. E. Sobolevskii and K. Van Lai, Difference schemes of optimal type for the approximate solution of parabolic equations (the Banach case), Ukrain. Mat. Zh. 33(1) (1981), 30-36.

[36] A. Ashyralyev and P. E. Sobolevskii, New Difference Schemes for Partial Differential Equations, Vol. 148 of Operator Theory: Advances and Applications, Birkhäuser Verlag, Basel, Boston, Berlin, 2004.

[37] A. Ashyralyev and P. E. Sobolevskii, On the stability of the delay parabolic differential and difference equations, Abstr. Appl. Anal. 6(5) (2001), 267-297.

[38] Yu. A. Smirnitskii and P. E. Sobolevskii, Positivity of multidimensional difference operators in the C-norm, Uspekhi. Mat. Nauk 34(4) (1981), 202-201 (in Russian).

[39] Yu. A. Smirnitskii and P. E. Sobolevskii, Positivity of difference operators, in:Spline Methods, Novosibirsk, 1981 (in Russian).

[40] Yu. A. Smirnitskii and P. E. Sobolevskii, Pointwise estimates of the Green function of a difference elliptic operator, in: Vychisl. Methody Mekh. Sploshn. Sredy 15(4) (1982), 529-542 (in Russian).

[41] Yu. A. Smirnitskii and P.E. Sobolevskii, Pointwise estimates of the Green function of the resolvent of a difference elliptic operator with variable coefficients in $R^{n}$, Voronezh. Gosud. Univ. 1982, 32p. Deposited VINITI 5. 2. 1982, No. 3519 (in Russian). 
[42] P. E. Sobolevskii, On elliptic equations in a Banach space, Differensialnyye Uravneniya 4(7) (1969), 1346-1748 (in Russian).

[43] A. Ashyralyev and P. E. Sobolevskii, On a class two-step difference schemes of higher order of the accuracy for elliptic equations in a Hilbert space, in: Numerical Methods for the Solutions of the Transport Equations, Akad. Nauk Estonsk. SSR, Tartu(1990), 18-22 (in Russian).

[44] A. Ashyralyev and P. E. Sobolevskii, Well-posedness of the difference schemes of the high order of accuracy for elliptic equations, Discrete Dyn. Nat. Soc. 2006 (2006), Article ID 75153, 12 pages.

[45] L. M. Gershteyn and P. E. Sobolevskii, Well-posedness of the a Banach space, Differensialnyye Uravneniya 10(11) (1974), 2059-2061 (in Russian).

[46] L. M. Gershteyn and P. E. Sobolevskii, Well-posedness of the general boundary value problem for the second order elliptic equations in a Banach space, Differensialnyye Uravneniya 11(7) (1975), 1335-1337 (in Russian).

[47] S. I. Primakova and P. E. Sobolevskii, The coercive solvability of fourth order difference schemes, Differentsialnyye Uravneniya 10(9) (1974), 1699-1713 (in Russian).

[48] P. E. Sobolevskii, On the equations of the second order with a small parameter at the highest derivatives, Uspekhi. Mat. Nauk 19(6) (1964), 217-219 (in Russian).

[49] P. E. Sobolevskii, Difference Methods for the Approximate Solution of Differential Equations, Izdat. Voronezh. Gosud. Univ., Voronezh, 1975 (in Russian).

[50] P. E. Sobolevskii, On the well-posedness in $C$ of the first boundary value problem for difference elliptic and parabolic equations in rectangular domains, Vychisl. Tsentr Sibirsk. Otdel. Akad. Nauk SSSR Preprint, Novosibirsk, 1976, 10 pages (in Russian).

[51] P. E. Sobolevskii, Imbedding theorems for elliptic and parabolic operators in $C$, Dokl. Akad. Nauk SSSR 302(1) (1988), 34-37 (in Russian).

[52] P. E. Sobolevskii, Well-posedness of difference elliptic equations, Discrete Dyn. Nat. Soc. 1 (1997), 219-231.

[53] P. E. Sobolevskii and M.F. Tiunchik, On a difference method for approximate solution of quasilinear elliptic and parabolic equations, in: Trudy Nauchn. -Issled. Inst. Mat. Voronezh. Gos. Univ. No. 2(1970), 82-106. (in Russian).

[54] P. E. Sobolevskii and M. F. Tiunchik, The difference method of approximate solution for elliptic equations, in: Trudy Nauchn. -Issled. Inst. Mat. Voronezh. Gos. Univ. No. 4(1970), 117-127 (in Russian).

[55] P. E. Sobolevskii and V. A. Pogorelenko, Hyperbolic equations in Hilbert space, Sibirskii Matematicheskii Zhurnal 8(1) (1967), 123-145 (in Russian).

[56] P. E. Sobolevskii and L. M. Chebotaryeva, Approximate solution by method of lines of the Cauchy problem for an abstract hyperbolic equations,mIzv.Vyssh.Uchebn. Zav.,Matematika 5 (1977), 103-116 (in Russian). 
[57] A. Ashyralyev and P. E. Sobolevskii, A note on the difference schemes for hyperbolic equations, Abstr. Appl. Anal. 6(2) (2001), 63-70.

[58] A. Ashyralyev and P. E. Sobolevskii, On the two new approaches of the high order of accuracy difference schemes for the second order differential equations, Functional Differential Equations 10(3-4) (2003), 333-405.

[59] A. Ashyralyev and P. E. Sobolevskii, Two new approaches for construction of the high order of accuracy difference schemes for hyperbolic differential equations, Discrete Dyn. Nat. Soc., 2005(2) (2005), 183-213.

[60] V. Pogorelenko and P. E. Sobolevskii, The "counter-example" to W. Littman counterexample of Lp -energetical inequality for wave equation, Functional Differential Equations 4(1-2) (1997), 165-172.

[61] S. Semenov and P. E. Sobolevskii, On some approach to investigation of singular hyperbolic equations, Dokl. Akad. Nauk SSSR 270(1) (1983), 555-558 (in Russian).

[62] A. Ashyralyev, G. Judakova and P. E. Sobolevskii, A note on the difference schemes for hyperbolic-elliptic equations, Abstr. Appl. Anal. 2006 (2006), Article ID 14816, 13 pages.

Received December 31, 2018; revised December 10, 2019; accepted December 15, 2019.

\section{Author information}

Allaberen Ashyralyev, Department of Mathematics, Near East University, Nicosia, TRNC, Mersin 10, Turkey \& Peoples' Friendship University of Russia (RUDN University), Moscow 117198, Russia \& Institute of Mathematics and Mathematical Modeling, 050010, Almaty, Kazakhstan.

E-mail: allaberen.ashyralyev@neu.edu.tr

Abdullah S. Erdogan, Valencia College, Orlando, FL, 32826, USA.

E-mail: aserdogan@gmail.com 